\title{
Phase-Stabilized Ammonium Nitrate-Based Propellants Using Binders with N-N Bonds
}

\author{
Charlie Oommen* and Sampat R. Jain ${ }^{\dagger}$ \\ Indian Institute of Science, Bangalore 560 012, India
}

\begin{abstract}
Phase-stabilized ammonium nitrate- (PSAN-) based solid propellants have been cast using new epoxy resins having $\mathrm{N}-\mathrm{N}$ bonds in their structures, as binders. The burning rate studies carried out in a strand burner show that significant enhancement in the burning rate, compared with that observed with conventional polybutadiene binders, could be achieved by the use of the N-N-bonded binders. The PSAN-based propellants have a higher burning rate than those based on ammonium nitrate. The enhancement in burning rate cannot be explained in terms of the calorific value of the resin or the ignition temperature of the propellant. The computed performance parameters of the compositions having $\mathrm{N}-\mathrm{N}$-bonded resins as binders are superior to those based on polybutadienes. Significantly higher specific impulse and chamber temperature obtained at practical solid loadings appear to be an advantageous feature of the compositions having $\mathrm{N}-\mathrm{N}$-bonded resins as binders.
\end{abstract}

\author{
Nomenclature \\ $C^{*}=$ characteristic velocity, $\mathrm{m} / \mathrm{s}$ \\ $I_{\mathrm{sp}}=$ specific impulse, $\mathrm{s}$ \\ $M_{c}=$ mean molecular weight of product species \\ $P_{c}=$ chamber pressure, atm \\ $P_{e}=$ exit pressure, atm \\ $T_{c} \quad=$ chamber pressure, $\mathrm{K}$ \\ $\Phi=$ equivalence ratio
}

\section{Introduction}

$\mathbf{O}$ NE of the few serious drawbacks of commonly used ammonium perchlorate- (AP-) based solid propellants is that their products of combustion, which include $\mathrm{HCl}$, chlorine, and chlorine oxides, cause atmospheric pollution. Not only do they affect the launch site surroundings, but these products are also known to cause ozone depletion in the atmosphere. Increasing concern for a clean atmosphere in recent years has resulted in a compelling need for developing chlorine-free, environmentally friendly, smokeless propellant systems. Various attempts made in this directioninclude the use of nitramines such as cyclotetramethylenettranitramine (HMX), cyclotrimethylenetrinitramine (RDX), etc., instead of AP; the inclusion of $\mathrm{Mg}$ in $\mathrm{AP}$ propellants to scavenge $\mathrm{HCl}$; the use of other oxidizers such as ammonium dinitramide (ADN); and ammonium nitrate (AN), etc., in the propellant composition..$^{1-4}$ These attempts have led to a rejuvenatedinterest in AN-based propellants, primarily because of the clean burning nature of AN, its low cost, and easy availability. However, although AN is well known as a low-energy oxidizer in gas generator compositions, its use in large motors is restricted as a result of several of its major adverse characteristics. Its hygroscopicity and near-room-temperaturephase transformation resulting in volume expansion cause storage problems and lead to crack formation in the grain. These problems, of late, have largely been overcome. Phase stabilization has been achieved by cocrystallizing AN with $\mathrm{K}^{+}$salts and other additives. ${ }^{5-10}$ The stabilization of phase also improves upon its hygroscopicity, which can more efficiently be managed by coating the particles with hydrocarbon/ polymers, etc. The other major problems in the use of AN as a substitute oxidizer for AP in solid propellants are its low burning rate

Received 17 January 1998; revision received 12 October 1998; accepted for publication 14 December 1998. Copyright $(\mathcal{O} 1999$ by Charlie Oommen and Sampat R. Jain. Published by the American Institute of Aeronautics and Astronautics, Inc., with permission.

${ }^{*}$ Scientific Officer, Department of Aerospace Engineering.

${ }^{\dagger}$ Professor Emeritus, Department of Aerospace Engineering.
(BR) and low energy. The AN-based propellants processed with the usual hydrocarbon binders burn at nearly one-fourth the rate of AP-based propellants. Different approaches adopted to enhance the BR of AN-based propellants include the use of catalysts such as chromium compounds, ${ }^{11}$ the addition of metals such as $\mathrm{Mg}^{4}$, the use of a high BR oxidizer such as AP, ${ }^{12}$ and energetic binders based on azide polymers. ${ }^{13}$ Of these, the approach based on energetic binders seems to be the most promising. However, poor ignitability, combustion instability, and heavy plasticization required for achieving adequate mechanical properties are some of the drawbacks of the azide-polymer-based systems. ${ }^{13,14}$

In our laboratory, a recent development in propellant binders has been the synthesis of hydrazine-based (having N-N bonds in the structure) viscous resins having epoxy end groups. ${ }^{15}$ Although initially these resins were synthesized for their use as binders for powder fuel compositions for hypergolic hybrid propellant systems, ${ }^{16}$ it was envisaged that these may augment the burning rates of solid propellants. Indeed, the AP-based propellants processed with $\mathrm{N}-\mathrm{N}$-bonded binders were found to have enhanced burning rates; almost three- to fourfold higher ${ }^{17}$ compared with those processed with the usual carboxyl-terminated polybutadiene (CTPB) binder. A sizable enhancement in the burning rate of AN-based propellants was also noticed in a recent study. ${ }^{18}$

Propellants having a stoichiometric fuel/oxidizer ratio are preferred for the maximization of combustion energy (specific impulse). This, however, is not achieved when a butadiene binder is used because of the solid loading limitation for processing. With the maximum solid loading possibility being limited to about $86 \%$ solids, most solid propellants based on polybutadiene binders remain fuel rich and do not yield the expected theoretical specific impulse $I_{\mathrm{sp}}$ in practice. In this sense, the binders having $\mathrm{N}-\mathrm{N}$ bonds could be advantageous because of the higher requirement of the binder (fuel) content for stoichiometric combustion.

Because AN as such, cannot be used in a propellant composition as a result of problems associated with the room-temperature phase transformation, phase-stabilized ammonium nitrate (PSAN) was used in the present study. In this paper we report the effects of several typical N-N-bonded resins based on carbono- and thiocarbono-hydrazones with epoxy end groups, on the burning rate of PSAN-based propellants. These data have been compared with propellants based on CTPB rather than a hydroxy-terminated polybutadiene(HTPB) binder, which is known to give slightly better performance. However, the processing of laboratory-scale propellants for burning rate was found to be easier with CTPB. The theoretical rocket performance parameters such as $I_{\mathrm{sp}}, T_{c}, C^{*}$, and $M_{c}$ of these systems have also been evaluated at various compositions to assess the effect of the new binders. 